\title{
A perspective on the challenges and issues in developing biomarkers for human allergic risk assessments
}

\author{
“...reliable and robust complementary biomarkers as a panel with \\ validated sensitivity and specificity need to be developed..."
}

First draft submitted: 24 April 2017; Accepted for publication: 9 May 2017;

Published online: 12 July 2017

Keywords: allergic response $\bullet$ biomarker $\bullet$ drug $\bullet$ immunity $\bullet$ medical device

Homeostasis in host immunity is the functional state that protects the body from disease while minimizing detrimental effects of an immune response, such as allergic, autoimmune or a suppressed immune response. As the normal human population's immunity can vary by more than two standard deviations [1-4], individuals can have wide fluctuations in how they express homeostasis.

When these possible detrimental effects are considered, immunotoxicity or immune disorders can be divided into two categories: enhanced immunity (e.g., hypersensitivity, allergy, autoimmunity, sensitization and dermatitis) and suppressed immunity (e.g., hyposensitivity and immunosuppression), shown in Figure 1. Both categories represent an immune response that is unable to recognize self from nonself appropriately - presenting a risk such as an allergic response [1].

\section{Exposure to an allergen}

Although the allergenicity of a substanceinduced allergic response is highly subjectspecific, a dose of allergen exceeding the threshold of a subject-specific level can cause an allergic response. Usually the dose is lower than a general toxicity level, but high enough to perturb the host immune system's homeostasis. This can occur through a single exposure or through multiple exposures. The first exposure might not cause any noticeable effects and some metal sensitizations can be triggered in humans by another metal, such as palladium cross reacting with nickel [5]. Once an allergen interacts with the individual, the immune system undergoes antigen processing to form a subject and allergen-specific hapten that can be processed and presented by antigen presenting cells. The response initiates an activation and maturation process, allowing the host immune system to memorize the 'signature', or the structured pattern, of the allergen complex. This first exposure can also adjust the threshold for a response. If a second exposure takes place, the immune system then quickly recognizes it as nonself and seeks to eliminate it in order to maintain the body's homeostasis. The protective action of the immune response is a doubleedged sword; it removes the perceived threat, but it can result in damage to the host locally or systemically, ranging from pain to serious illness including death from anaphylaxis [1]. Here, we focus on metal and drug-associated allergic risks.

\section{Response to an allergen}

The response to an allergen is complicated as it depends on an individual's immune system. In other words, the same exposure could have diverse results in a population of individuals and an individual's response can change during a lifetime [6]. In contrast, general toxicity is a dose-dependent event that shows relatively uniform manifestations from one individual to another. Allergic reactions are historically classified as type I, II, III and IV, but the mercury in dental amalgams can
Ying Mu*,1, Dianne E Godar ${ }^{1}$ \& Stephen J Merrill ${ }^{2}$ ${ }^{1}$ Center for Devices \& Radiological Health, U.S. Food \& Drug Administration, 10903 New Hampshire Ave., Silver Spring, MD 20993, USA

2Department of Mathematics, Statistics \& Computer Science, Marquette University, Milwaukee, WI 53201-1881, USA

*Author for correspondence:

+1 7247593066

yingm6@gmail.com 


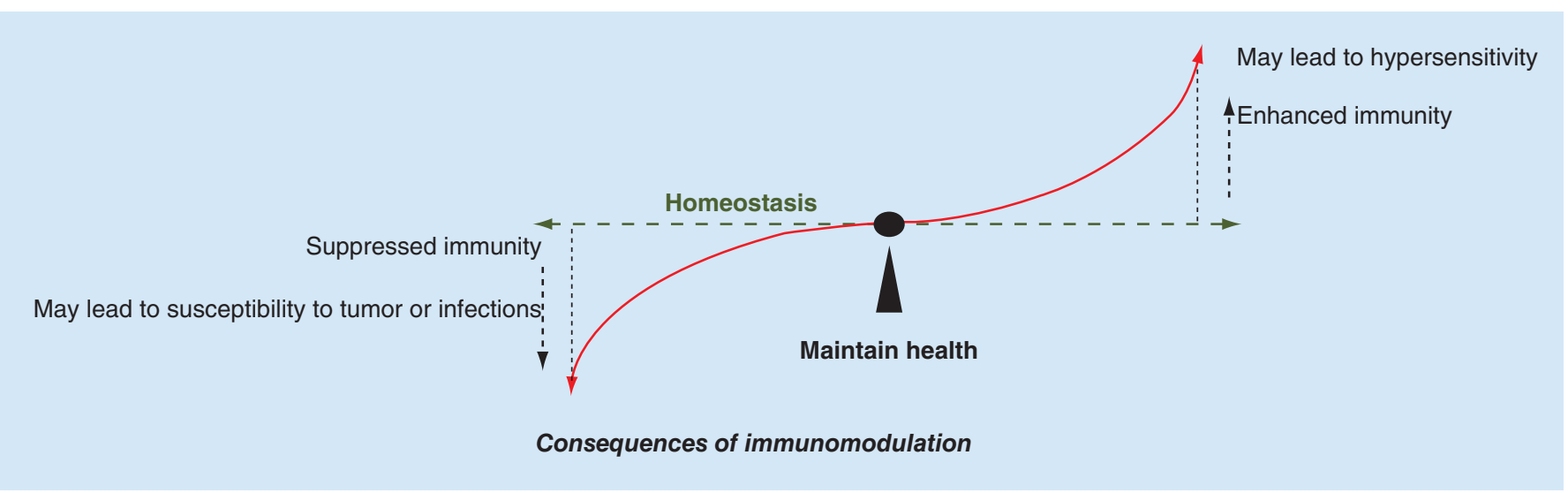

Figure 1. A model of immunomodulation in homeostasis.

induce all types of allergic responses [7,8]. While nickel causes a type IV lymphocyte mediated delayed-type allergic response, it displays a common inflammatory morphological pattern, observed as a regional lymphocyte infiltration also seen in many other instances such a viral infection $[9,10]$. This challenges and confounds diagnostic and monitoring efforts that have an impact on clinical decision-making [11]. In the clinical setting, the manifestations usually do not show a clear cutoff between the different types of allergic responses, because the inflammatory response can be similar while the allergic response can be mixed [12-14]. We propose considering an additional category that is based on a substance classification, such as 'metals' to cover mercury and nickel allergies rather than a type based, to provide a clearer picture in clinical decision-making.

Biomarkers are commonly used as diagnostic and monitoring tools that offer distinct advantages for improving clinical outcome [15]. Uncovering biomarkers to stratify the allergic risks and streamline clinical information can have a positive effect on medical interventions [16,17]. Ideally, physicians could then predict personalized risk prior to introduction of a metal-containing medical device or a drug [17].

\section{Current challenges \& issues}

Stratification of categories associated with the allergens might result in a better understanding of the mechanisms behind the phenomena. Currently preclinical allergic risk assessments rely on animal-based tests [18]. However, this approach has been criticized for providing poor clinical relevance due to species variation in immune responses and drug metabolism [19-21]. Among animal species, nonhuman primates are considered the closest model for representing the human situation. However, sometimes human and nonhuman primates display a significant difference in their immune response, for example, their reaction to herpes simplex viral infection $[4,20,22]$. In drug-induced allergic responses, metabolites of a drug can also result in a specific allergic response in humans that is different from what is observed in nonprimate humans and can even be specific to an individual due to their unique forms of metabolic enzymes. Consequentially, the metabolite-hapten complex formation through human antigen presenting cells might also be unique. In devices that contain metals, nickel is a well-known human allergen with an allergic response prevalence estimated between 17 and $20 \%$ in the general population; but it is not an allergen in the mouse due to differences in Toll-like receptor 4 [23-25].

Nickel, cobalt and chromium metals can leach out from alloy-based medical devices and 'wear particles' can form and increase with time from common daily activities. High concentrations of salts and amino acids along with mechanical loading, the interface between the device and the tissues, and the battery effects between dissimilar metals all promote metal ion release from a device. Patients who have allergic reactions commonly have high blood levels of metals [26]. Metal ions need to be conjugated with proteins to be recognized by a host immune system. In the case of nickel, the reconfirmation of endogenous proteins with nickel enlarges their size and the result can be allergenic $[27,28]$. However, these alloys must be used because they provide the mechanical strength necessary to support the body part, for example, the metallic hip joint supports the leg. Another concern is that computational modeling of structure-activity relationship and animal data do not discern between those who will get the product-related allergic response and those who will not [29,30], but both are somewhat informative for weighting risks and benefits.

Because funding sources are more concerned with addressing major diseases than developing allergic biomarkers, the ones currently used in the clinics are 
considered obsolete [31]. As an alternative method, the human lymphocyte transformation test is considered as the secondary tier of tests for showing clinical relevance $[32,33]$. Recently, environmentally friendly lymphocyte transformation tests were developed using fluorescent probes and flow cytometry to eliminate the need of radioactive isotopes and to offer more precise information [34].

Commonly used animal-based testing models do not always predict the human outcome in a clinical study. This points out the importance of performing clinical trials [35], because some adverse events can only manifest from human biochemical and immunological reactions. For example, drugs repeatedly associated with autoimmune responses can cause drug-induced liver injury. Although only one out of 1000 to 10,000 humans experience drug-induced liver injury from resulting autoantibodies, irreversible liver failure is possible. Predicting which drugs will cause injury is an inexact science and monitoring alanine aminotransferase is an ineffective approach outside of clinical trials because it lacks specificity and sensitivity [36]. Allergic responses occur at a lower frequency in the clinic because they can take a long time to manifest; sometimes mild-sickness and nondisease-specific symptoms of acute liver damage can take a few months or longer to develop after taking the medication. Without appropriate biomarkers, it is challenging to weight the risks and benefits accurately [37,38]. Thus, many case reports and adverse events were apparently overlooked due to the lack of availability of appropriate biomarkers [26] and it is estimated that allergic-related adverse events may have been under reported by as much as $90 \%$ [38].

Lack of communication can exist between the immunologists (allergist) and the surgeons or dentists who implant these metal containing devices and the physicians who prescribe the drugs. Even in the allergist setting, there is a lack of robust and

\section{References}

1 Musa M. Immune mechanism: a 'double-edged sword'. Malays. J. Med. Sci. 20(3), 61-67 (2013).

2 Gustafson MP, Lin Y, Ryder M, Dietz AB. Strategies for improving the reporting of human immunophenotypes by flow cytometry. J. Immunother. Cancer 2, 18 (2014).

3 Mcloughlin K, Turteltaub K, Bankaitis-Davis D et al. Limited dynamic range of immune response gene expression observed in healthy blood donors using RT-PCR. Mol. Med. 12(7-8), 185-195 (2006).

4 Luebke RW, Parks C, Luster MI. Suppression of immune function and susceptibility to infections in humans: association of immune function with clinical disease. J. Immunotoxicol. 1(1), 15-24 (2004). reliable test methods. For example, skin patch and intradermal tests often show large numbers of false positives and negatives [39]. Once positive to an allergen such as nickel, the positivity will remain for a very long time and possibly for the individual's entire life, which does not reflect the clinical situation when the implant was removed and the symptoms no longer exist, but the test continues to remain positive [26]. Furthermore, these tests cannot differentiate between palladium cross-reacting as nickel, because palladium has $90 \%$ cross reactivity with nickel [5].

In conclusion, to prevent allergic risks by capturing measurable signals of allergy in hypersensitive individuals and to improve diagnosing and monitoring patients' health, clinically relevant, reliable and robust complementary biomarkers as a panel with validated sensitivity and specificity $[36,40]$ need to be developed using relevant human material combined with individual-based in vitro test models to accurately predict the long-term clinical outcome $[35,41]$.

\section{Disclaimer}

The points of view in this article have not been formally disseminated by the US FDA and should not be considered to represent any agency determination or policy.

\section{Release of copyright permission}

Written products prepared by Federal employees as part of official duties are in the public domain and as such, there is no assignment of copyright (17 U.S.C. 105).

\section{Financial \& competing interests disclosure}

Y Mu and DE Godar are US Government employees. The authors have no other relevant affiliations or financial involvement with any organization or entity with a financial interest in or financial conflict with the subject matter or materials discussed in the manuscript apart from those disclosed.

No writing assistance was utilized in the production of this manuscript.

5 Hindsen M, Spiren A, Bruze M. Cross-reactivity between nickel and palladium demonstrated by systemic administration of nickel. Contact Dermatitis 53(1), 2-8 (2005).

6 Poole CJ. Illness deception and work: incidence, manifestations and detection. Occup. Med. (Lond.) 60(2), 127-132 (2010).

7 Mcgivern B, Pemberton M, Theaker ED, Buchanan JA, Thornhill MH. Delayed and immediate hypersensitivity reactions associated with the use of amalgam. Br. Dent. J. 188(2), 73-76 (2000).

8 Anneroth G, Ericson T, Johansson I et al. Comprehensive medical examination of a group of patients with alleged adverse effects from dental amalgams. Acta Odontol. Scand. 50(2), 101-111 (1992). 
$9 \quad \mathrm{Ng}$ VY, Lombardi AV Jr, Berend KR, Skeels MD, Adams JB. Perivascular lymphocytic infiltration is not limited to metal-on-metal bearings. Clin. Orthop. Relat. Res. 469(2), 523-529 (2011).

10 Feng Q, Wei H, Morihara J et al. Th2 type inflammation promotes the gradual progression of HPV-infected cervical cells to cervical carcinoma. Gynecol. Oncol. 127(2), 412-419 (2012).

11 El-Mawardy R, Fuad H, Abdel-Salam Z, Ghazy M, Nammas W. Does nickel allergy play a role in the development of in-stent restenosis? Eur. Rev. Med. Pharmacol. Sci. 15(11), 1235-1240 (2011).

12 Forte G, Petrucci F, Bocca B. Metal allergens of growing significance: epidemiology, immunotoxicology, strategies for testing and prevention. Inflamm. Allergy Drug Targets 7(3), 145-162 (2008).

13 Schram SE, Warshaw EM, Laumann A. Nickel hypersensitivity: a clinical review and call to action. Int. J. Dermatol. 49(2), 115-125 (2010).

14 Thomas P, Thomsen M. [Implant allergies]. Hautarzt 61(3), 255-262; quiz 263-254 (2010).

15 Biomarkers as Predictive Tools. http://staging-ecvam.jrc.it/

16 Sargent DJ, Conley BA, Allegra C, Collette L. Clinical trial designs for predictive marker validation in cancer treatment trials. J. Clin. Oncol. 23(9), 2020-2027 (2005).

17 Qamar A, Bernstein LH, Zarich S. Outcomes research is the fifth discipline of the fifth generation of managed care: utility of a single troponin-T. Clin. Lab. Manage. Rev. 12(2), 80-86 (1998).

18 Anderson SE, Siegel PD, Meade BJ. The LLNA: a brief review of recent advances and limitations. J. Allergy (Cairo) 2011, 424203 (2011).

19 LLNA NIH Publication Number 11-7709. https://ntp.niehs.nih.gov/

20 Huff JL, Barry PA. B-virus (Cercopithecine herpesvirus 1) infection in humans and macaques: potential for zoonotic disease. Emerg. Infect. Dis. 9(2), 246-250 (2003).

21 Evans DT, Silvestri G. Nonhuman primate models in AIDS research. Curr. Opin. HIV AIDS 8(4), 255-261 (2013).

22 Nguyen DH, Hurtado-Ziola N, Gagneux P, Varki A. Loss of Siglec expression on T lymphocytes during human evolution. Proc. Natl Acad. Sci. USA 103(20), 7765-7770 (2006).

23 Schmidt M, Raghavan B, Muller V et al. Crucial role for human Toll-like receptor 4 in the development of contact allergy to nickel. Nat. Immunol. 11(9), 814-819 (2010).

24 Zoroddu MA, Peana M, Medici S, Potocki S, Kozlowski $\mathrm{H}$. Ni(II) binding to the 429-460 peptide fragment from human Toll like receptor (hTLR4): a crucial role for nickelinduced contact allergy? Dalton Trans. 43(7), 2764-2771 (2014).

25 Rachmawati D, Bontkes HJ, Verstege MI et al. Transition metal sensing by Toll-like receptor- 4 : next to nickel, cobalt and palladium are potent human dendritic cell stimulators. Contact Dermatitis 68(6), 331-338 (2013).

26 Amini M, Mayes WH, Tzeng A, Tzeng TH, Saleh KJ, Mihalko WM. Evaluation and management of metal hypersensitivity in total joint arthroplasty: a systematic review. J. Long. Term Eff. Med. Implants 24(1), 25-36 (2014).

27 Grammer LC, Shaughnessy MA, Patterson R. Modified forms of allergen immunotherapy. J. Allergy Clin. Immunol. 76(2 Pt 2), 397-401 (1985).

28 Heydenreich B, Bellinghausen I, Lund L et al. Adjuvant effects of aluminium hydroxide-adsorbed allergens and allergoids - differences in vivo and in vitro. Clin. Exp. Immunol. 176(3), 310-319 (2014).

$29 \mathrm{Hu}$, Hu Z, Zhang Y et al. Metal binding mediated conformational change of XPA protein: a potential cytotoxic mechanism of nickel in the nucleotide excision repair. J. Mol. Model 22(7), 156 (2016).

30 Ouyang Q, Wang L, Mu Y, Xie XQ. Modeling skin sensitization potential of mechanistically hard-to-beclassified aniline and phenol compounds with quantum mechanistic properties. BMC Pharmacol. Toxicol. 15, 76 (2014).

31 Maxwell G, Aeby P, Ashikaga T et al. Skin sensitisation: the Colipa strategy for developing and evaluating non-animal test methods for risk assessment. ALTEX 28(1), 50-55 (2011).

32 Thomas P, Von Der Helm C, Schopf C et al. Patients with intolerance reactions to total knee replacement: combined assessment of allergy diagnostics, periprosthetic histology, and peri-implant cytokine expression pattern. BioMed Res. Int. 2015, 910156 (2015).

33 Whritenour J, Ko M, Zong Q et al. Development of a modified lymphocyte transformation test for diagnosing drug-induced liver injury associated with an adaptive immune response. J. Immunotoxicol. 14(1), 31-38 (2017).

34 Dziubek Z, Zarnowska H, Basiak W, Gorski A, Kajfasz P. [Some aspects of immune response in toxoplasmosis]. Przegl. Epidemiol. 55(4), 495-502 (2001).

35 Faris O, Shuren J. An FDA viewpoint on unique considerations for medical-device clinical trials. N. Engl. J. Med. 376(14), 1350-1357 (2017).

36 Delemos AS, Foureau DM, Jacobs C, Ahrens W, Russo MW, Bonkovsky HL. Drug-induced liver injury with autoimmune features. Semin. Liver Dis. 34(2), 194-204 (2014).

37 Ahmad J, Odin JA. Epidemiology and genetic risk factors of drug hepatotoxicity. Clin. Liver Dis. 21(1), 55-72 (2017).

38 Jungst C, Graber S, Klahn D, Wedemeyer H, Lammert F. [Prevalence and risk factors of drug induced liver disease: a survey based study in pharmacies]. Z. Gastroenterol. 54(2), 131-138 (2016).

39 Zaghi D, Maibach HI. Quantitative relationships between patch test reactivity and use test reactivity: an overview. Cutan. Ocul. Toxicol. 27(3), 241-248 (2008).

40 Martin AK, Malhotra AK, Sullivan BL, Ramakrishna H. Troponin elevations in patients with chronic cardiovascular disease: an analysis of current evidence and significance. Ann. Card. Anaesth. 19(2), 321-327 (2016).

41 Huang JX, Blaskovich MA, Cooper MA. Cell- and biomarker-based assays for predicting nephrotoxicity. Expert Opin. Drug Metab. Toxicol. 10 (12), 1621-1635 (2014). 